\title{
33. COMMISSION DE LA STATISTIQUE STELLAIRE
}

Président: M. J. H. Oort.

Membres: MM. Ambartsumian, Baade, R. H. Baker, Blaauw, Blanco, Bok, Bourgeois, Camm, Chandrasekhar, Delhaye, Durand, Dziewulski, Elvius, Finlay-Freundlich, Fricke, Gratton, Habibulin, Heckmann, Hertzsprung, Ikaunieks, Junkes, Kaburaki, Kuiper, Kukarkin, Lindblad, Lindsay, Lundmark, Luyten, Lyttkens, McCuskey, MacRae, Malmquist, Mergentaler, F. D. Miller, Mineur $\dagger$, Mohr, W. W. Morgan, Nassau, Neyman, Nordström, Odgers, Ogorodnikov, Pannekoek, Parenago, Perek, Ramberg, Reiz, Mlle Roman, MM. Safronov, Schalén, Schilt, Schürer, H. Shapley, Smart, Trumpler $\dagger$, Vanderlinden, van Hoof, van Rhijn, Vashakidze $\dagger$, Vyssotsky, Mme Williams-Vyssotsky, M. Weaver.

\section{3a. Sous-Commission pour la CoORdination des Recherches Galactigues}

Président: M. J. H. Oort.

Membres: MM. Baade, Blaauw, Lindblad, Parenago, van Rhijn.

\section{INTRODUCTION}

The domain of this commission is wide and overlaps that of several other commissions. Some limitations had therefore to be set in preparing the following report. In the first place it has been limited, like the report presented at the Rome meeting, to some problems and investigations of the past three or four years that appeared to me most important for our insight into the structure and dynamics of the Galactic System. But even within these bounds it is very incomplete, for which I offer my apologies.

Great advances have been made in recent years, in the first place through the successful observations of the radiation at $2 \mathrm{I} \cdot \mathrm{I} \mathrm{cm}$. wave-length emitted by interstellar hydrogen, in the second place through extended surveys of very distant stars and nebulae in the Galactic System. In the few years which have elapsed since the writing of the preceding report, these observations have already revealed a large part of the spiral structure of this System, and have given us important information on the mass distribution.

Side by side another development, no less fundamental for the subject of this commission, has made large forward strides, namely, observations and theory bearing on the evolution of stars. These topics will not be discussed, as they were considered to fall outside the scope of Commission 33.

During the period to be reviewed two general books dealing with the subject of galactic structure and dynamics were published: B. W. Kukarkin, Erforschung der Struktur und Entwicklung der Sternsysteme auf der Grundlage des Studiums veränderlicher Sterne (Akademie-Verlag, Berlin, I954) and R. J. Trumpler and H. F. Weaver, Statistical Astronomy (University of California Press, I953). The former is a translation of a book published in Russian in 1949; here and there references to more recent investigations have been added.

\section{SPIRAL STRUCTURE}

The first definite evidence of spiral structure in the Galactic System was discovered in I95I by Morgan, Sharpless and Osterbrock(r). They found that the great emission regions were confined to two 'arms', one passing through the Sun, the other through the double cluster in Perseus. Since then, several investigators have shown that $O$ associations and blue supergiants in general lie in spiral arms (z). Miss Torgård (3) has shown that $\delta$ Cephei variables are concentrated in the same arms. Guido Münch obtained spectra in which the interstellar absorption lines gave clear evidence of the two arms mentioned above (4). 
The largest amount of information on the spiral structure has, however, been obtained from measures of the hydrogen emission line at. $2 I \mathrm{~cm}$. wave-length $(5,6)$. The observations published so far give mainly the distribution of hydrogen atoms in the galactic plane for distances from the centre greater than that of the Sun. The longitude interval covered extends from $320^{\circ}$ to $220^{\circ}$. Observations in the southern Milky Way are being made in Australia by Kerr, Hindman and Robinson. A new series of observations at Kootwijk, Netherlands, covers more fully the galactic circle from $320^{\circ}$ to $220^{\circ}$ and includes at every $5^{\circ}$ longitude measures at various latitudes from $-I^{\circ}$ to $+I^{\circ}$ (except for longitudes below $45^{\circ}$, where they extend from $-6{ }^{\circ}$ to $+5^{\circ} 5$ ). This will be completed in the spring, I955. Reductions for the inner parts of the System are now in progress. It has already been possible to determine the effective thickness of the hydrogen layer in the whole of the inner region of the Galactic System observable from the Netherlands (6); this was found to vary only a little. The average is about 240 ps, which is very close to the thickness found in the vicinity of the Sun. Also, parts of inner arms could be traced provisionally.

The evidence so far obtained indicates that the galactic spiral rotates such that the convex sides of the arms precede. The observation of spiral arms in the Galactic System, where the differential rotation is known and strong, emphasizes the problem of the origin and continued existence of these arms. With an average spacing of about $2 \mathrm{kps}$ and an average derivative of angular velocity with respect to distance from the centre of $-9.5 \mathrm{~km}$./sec.kps, the present arms would rapidly dissolve, unless they be constantly rejuvenated by a process not yet understood. Without such a process they could not be older than 300 million years.

Apparently, gas plays an important part in the spiral arms. It appears even probable that gas is the primary constituent, and that the stars we find concentrated in the arms are only a byproduct. These results suggest that it may be the specific properties of the gas that give rise to the spiral structures. In what way they do this is still obscure. One can understand why the gas has a tendency to contract to a flat disk, as it has done in the Galactic System. One can similarly understand that the differential angular rotation will draw out all irregularities into spiral or circular bands. But the large-scale structures extending over the whole system remain as yet unexplained.

For a better insight into the spiral structure, observations of the $2 \mathrm{I} \mathrm{cm}$. line and stellar observations will both be needed. The former can give a comprehensive picture, but are restricted to the measurement of velocities and intensities, from which distances and densities can only be inferred indirectly, by assuming the gas to move in a preconceived way (e.g. in circular orbits). In the region inside the Sun's distance from the centre even this is difficult, because there are always two regions having the same velocity. By observing supergiants, of which distances can be computed without reference to velocities, we can obtain a less hypothetical picture of the arms, and, moreover, study possible systematic deviations from circular motion. The two kinds of observations can supplement each other in a very desirable manner.

Thus far, the observations of supergiants have mostly been restricted to relatively small parts of the spiral arms in the general vicinity of the Sun. Because of the absorption it will probably be impossible to observe early-type supergiants or $\delta$ Cephei variables in any completeness over the entire system. However, recent advances in the technique of discovering early-type supergiants to a rather faint limit hold out a promise that in relatively transparent regions it may soon be possible to find such stars over perhaps as much as half the extent of the Galactic System (cf. the next section). A promising way of locating the spiral arms is also the discovery of faint emission nebulae; this is then to be followed up by a study of the stars involved in them. For the southern hemisphere such studies have been commenced by Bok and collaborators. High-resolution surveys at short radio wave-lengths may likewise contribute to the discovery of distant emission nebulae. A first step has been made with the 50-foot dish of the Naval Research Laboratory in Washington, where, on a wave-length of $9.4 \mathrm{~cm}$., several emission nebulae could be located ( ) . 
Surveys for faint $\delta$ Cephei variables are being continued at various observatories, but they are extremely laborious. Great progress in the accurate location of $\delta$ Cephei variables in space will be made when the extensive Lick and Leiden-Johannesburg programmes on photo-electric colours are completed. A new determination of the zero point of the period-luminosity relation, using newly determined proper motions of the bright objects, was made by Blaauw and Morgan (8). An important programme of radial velocities of 55 southern $\delta$ Cephei variables has been carried out by Stibbs with the Radcliffe reflector at Pretoria.

Radial velocities of $\mathrm{I}_{78}$ southern $\mathrm{O}-\mathrm{B}_{5}$ stars down to $7^{\mathrm{m}} \cdot 5$ have been completed at the Radcliffe Observatory, and are being prepared for publication. These observations will be extended to supergiants of fainter apparent magnitude. OB stars down to I0.6 photographic magnitude are being observed for radial velocity at the David Dunlap Observatory.

The data on colour excesses of southern $O$ and $B$ stars published by Oosterhoff in I95I (9) were extended by measures for I25 of such stars by Bok and van Wijk (1o).

These stars of extreme luminosity have presumably all been formed less than fifty million years ago. What they show, therefore, are the instantaneous spiral arms and their motions. If it were possible to identify a group of somewhat older stars, with ages of one or two hundred million years, and to obtain data on their distribution and motions in space, these might teach us something about the situation of spiral arms at the time when these stars were formed. For the motions of the stars must have been governed solely by the gravitational field, which, being largely due to the more central masses of the Galactic System, may in first approximation be treated as constant and determinable from present-day observations.

It is probable that A-type stars and those termed 'strong-line stars' by Miss Roman fulfil to some extent these conditions. Signs of large-scale irregularities in the distribution of $A$ and $F$ stars have already been found in several regions. Greater accuracy in the data and more extensive observations are wanted to confirm these. Statistical observations of A stars up to the largest distances attainable will evidently be of considerable importance. It is clear that, because these types of stars are very much fainter iritrinsically than the supergiants, observations would have to be pushed to faint limits, even to outline the nearest parts of spiral arms. The problem of how such observations would best be attempted was discussed extensively at a conference on Co-ordination of Galactic Research (see p. 484 below).

\section{SurveYs For Supergiants ANd M, N ANd S Stars}

One of the most promising tasks is the finding of distant stars in the galactic plane. In the preceding report $(\mathrm{Ix})$ mention has been made of several surveys of supergiant stars. These have since been considerably extended, in particular through work done at the Tonantzintla Observatory, where large numbers of new OB stars of high luminosity have been discovered (12). The studies at Tonantzintla also encompass a large part of the southern Milky Way; in all, 2r39 stars of spectral types $O$ and B were classified as high-luminosity objects. The Tonantzintla surveys are now being extended to the I3th $p g$ magnitude.

A new list of $27 \mathrm{O}$ associations was published by Morgan, Whitford and Code (r3). The same authors are preparing a catalogue of spectroscopic parallaxes of approximately rooo $\mathrm{O}$ and $\mathrm{B}$ stars of high luminosity. A large number of stars of this type have also been observed by Hiltner. Spectroscopic parallaxes for between 1500 and 2000 earlytype supergiants will probably be completed in I955.

Morgan and Meinel have developed a method for the discovery of very faint reddened early-type stars (14). It is hoped that a finding list of such objects to the I5th $p g$ magnitude will soon be practicable. Spectral criteria of absolute magnitude for high-luminosity stars are being studied at Stockholm Observatory, also with a view to low-dispersion work on faint stars. 
Considerable work has been done by Morgan, H. L. Johnson and Harris on the defining of a photometric system which will permit the most accurate determination of colour excesses and absorptions (15).

At the Warner and Swasey Observatory an infra-red survey of relatively cool stars has been completed in a region $4^{\circ}$ wide centred on the galactic equator, and extending in longitude from $333^{\circ}$ through zero to $20 \mathrm{r}^{\circ}$. The survey also covers a number of strips of $4^{\circ}$ width extending perpendicular to the Milky Way to latitudes of $\pm 18^{\circ}$. Most promising for the spiral-arm problem is a list of suspected supergiant $M$ stars (r6) and a catalogue of carbon stars (17). The stars of spectral class $\mathrm{M}_{5}$ and later, which constitute the bulk of the surveys just mentioned, give information on the smooth distribution of disk-population II objects in the Galactic System (18). The survey has now been extended to cover a $12^{\circ}$-wide zone within the same longitude limits. Fricke is using plates taken with the Cleveland Schmidt telescope to search for very distant M supergiants in the region from $20^{\circ}$ to $30^{\circ}$ longitude.

For the determination of the absolute magnitudes of the $\mathrm{M}$ stars the measurement of accurate proper motions is a first necessity. An extensive programme of proper motions of long-period variables is carried out at the McCormick Observatory, while also at Leiden plates have been taken for this purpose. Vyssotsky reports that measures have been completed for 286 of the 330 stars on the McCormick programme. This programme extends also over the southern hemisphere (southern plates were taken at the Yale Southern Station).

A survey of $M$ stars of different nature is the search for $M$ dwarfs on objective-prism plates which was recently completed by Vyssotsky at the McCormick Observatory. It has yielded $87 \mathrm{x}$ red dwarfs north of $-\mathrm{I} 5^{\circ}$ declination. For nearly all of them proper motions are now available. Dyer has determined radial velocities for 166 of these dwarfs from plates he took at the Mount Wilson Observatory.

Mention may be made of a useful catalogue of emission-line stars of types later than B which has been compiled by Bidelman (rg).

\section{Stellar Associations and B Stars}

Considerable work on associations has been done in the U.S.S.R., the U.S.A. and Mexico, giving extremely interesting results, for the so-called $\mathrm{O}$ associations as well as for the $T$ associations revealed by emission-line stars in regions of high interstellar density. However, this work will not be discussed here, as it was agreed that these subjects will be reported by other commissions.

Proper motions for 2I T Tauri stars in the Taurus dark clouds have been determined by Pels and Blaauw, and will soon be published.

The motions of the $\mathrm{O}$ and $\mathrm{B}$ stars in general were investigated in considerable detail by Torondzhadze (20) and by Blaauw. Both authors based their studies on the concept that the B stars have come from expanding associations. Blaauw's investigation, which has not yet been published, is confined to the nearest main-sequence B stars in the northern hemisphere and aims not only at the motions but likewise at a basic determination of the luminosities.

Weaver (2I) has indicated that in some regions the early B stars show a velocity dispersion that is much larger than the average for these stars.

\section{Young and Old Stars. Motions of the Relatively Young Stars. Star Streams. Stars of High Velocity}

It has become more and more evident that the common-type stars later than A consist of a mixture of relatively young and rather old stars. The most important contribution to this problem has been made by Miss Roman (22). Among the main-sequence stars of types $F_{5}-G_{5}$ as well as among the G8-KI giants and subgiants, she has found the 
possibility of distinguishing spectroscopically two large, and equally frequent groups, which show rather pronounced differences in velocity distribution. The two categories were termed 'strong-line' and 'weak-line' stars. In velocity distribution the former shows some resemblance to the A-type stars, the latter may represent a well-mixed disk population. Miss Roman's distinction has been confirmed from objective-prism spectra of faint $G$ and $K$ stars by Vyssotsky and Skumanich (23).

Another spectrographic distinction between stars of low and moderately high velocities was discovered independently by Delhaye and by Baade among the $M$ dwarfs. They found that the dwarfs with emission lines have very low average velocities, especially in the direction perpendicular to the galactic plane, indicating that these may be quite young stars (24).

Blaauw has made a study of the phenomenon of star streaming among the stars of Miss Roman's two groups. He found that, in particular for the G8III stars of the 'strong-line' type, the velocity vectors are not concentrated around zero velocity (after elimination of solar motion) as one would expect for an ellipsoidal distribution. They are concentrated in two regions centred around about $u=+25 \mathrm{~km}$. $/ \mathrm{sec}$., $v=+$ Io $\mathrm{km}$. $/ \mathrm{sec}$. and $u=-$ ro $\mathrm{km}$. $/ \mathrm{sec}$., $v=+5 \mathrm{~km}$. $/ \mathrm{sec}$., with internal velocity dispersion of about Io $\mathrm{km}$. $/ \mathrm{sec}$. ( $u$ towards $l=0^{\circ} b=0^{\circ}, v$ towards $\left.l=90^{\circ} b=0^{\circ}\right)$. The Ko III strong-line stars also show the concentration around the first-mentioned centre, but very few stars outside it. This investigation was inspired by earlier work done by Delhaye and collaborators.

It has been evident since the first investigations of the space motions of A- and early F-type stars that the velocity distributions of these stars of rather low velocity showed important irregularities. For the bright stars these can be partly correlated with the motion of the Ursa Major cluster and group and with the Taurus group, as was first shown by Strömberg. Delhaye (25) and Kovalevsky (26) have investigated local irregularities in the velocity distribution of near-by stars. They find that also for the stars of later types maxima are found in the velocity distribution which lie, respectively, in the general region of the Ursa Major stream, in that of the Taurus stream, and a third around the centre of the velocity distribution of the A stars.

Tannahill at Glasgow published a general investigation of star-streaming by means of the GC proper motions (27). He used Eddington's method and investigated three spectral groups in four zones of galactic latitude.

Miss Roman is making detailed spectrographic observations of all known non-variable stars of high velocity. Among the bright $\mathrm{G}_{5}-\mathrm{K}$ I stars she finds $5 \%$ stars with weak cyanogen bands. These stars have an average space velocity of $96 \mathrm{~km}$. $/ \mathrm{sec}$. From the nearly 600 high-velocity stars under investigation 17 stars were selected spectroscopically as 'intermediate white dwarfs'. These seem to be F-type stars with absolute magnitudes between +4 and +5 . The hydrogen lines are normal, but all other lines are very much weakened. A study of the space motions of these stars indicates that they move in highly eccentric galactic orbits with a mean eccentricity near 0.9 ; they penetrate into or near the galactic nucleus; they share only slightly or not at all in galactic rotation, and have as little concentration to the galactic plane as the RR Lyrae stars.

\section{General Investigations on Star Density and Absorption}

Several of the investigations reported on in the preceding report have been continued. At Cleveland the analysis of galactic structure has now been finished in nine Milky Way regions (28). While continuing his spectrophotometric magnitude and colour work down to $13^{\mathrm{m}} \cdot 5 \mathrm{pg}$ in selected fields in the Milky Way, Ramberg concentrated on the B and A stars, with a view to contributing to the question of the shape of the spiral structure in our surroundings. Elvius' spectrophotometric work is dealt with in the report of the Commission on Selected Areas. Spectrophotometric work at Uppsala has been concentrated on bright and dark regions in Taurus and Auriga, and has led to a better determination of the distances of the nebulae (29). Schalén has investigated general densities 
in two belts at $20^{\circ}$ and $40^{\circ}$ latitude, respectively (30), using spectra, colours and magnitudes down to II $\mathrm{m} \cdot \mathrm{o} p g$.

At Torun spectra, photographic and photovisual magnitudes are being determined for stars down to $13^{\mathrm{m}}$ in four galactic fields. Spectral plates have been supplied by the Warner and Swasey Observatory.

From Bruce plates Shapley has made a survey of dust clouds along the southern Milky Way, from longitudes $200^{\circ}$ to $340^{\circ}$ and between $-30^{\circ}$ and $+30^{\circ}$ latitude. One of the purposes is to find low-latitude galactic 'windows'. A similar outlining of dust clouds in the anti-centre region is nearly completed.

Of fundamental importance for the study of the general distribution of stars at large distances is the redetermination of the magnitude scales in ten of Kapteyn's Selected Areas, which has been carried out by Baum (still unpublished at the time of writing).

\section{Photo-electric Determinations of Spectral Classes and Luminosities}

The work done by Strömgren and Gyldenkerne on spectral classifications and spectroscopic luminosities with an accuracy far surpassing that which had been reached by photographic methods promises to open up a new aera for the determination of the density distribution of common-type stars, and for finding accurate distances and densities of interstellar clouds. As the subject will probably be treated in the report of Commission 29 or 36 , no details are given here.

\section{Work in High Latitudes. Density Distribution Perpendicular to The Galactic Plane}

Miss Roman has started a programme of slit spectra to classify stars down to $12^{\mathrm{m}} \cdot \mathrm{o} p \mathrm{~g}$. in a number of Selected Areas within $40^{\circ}$ of the galactic poles. In a large number of Selected Areas in these regions she is determining spectra for the so-called fundamental stars (see report of Commission 32). At the David Dunlap Observatory it is intended to determine radial velocities of part of the stars in this programme.

$\mathrm{Mr} \mathrm{Hill}$, at Leiden, has made an extensive investigation of the distribution of the proper motions, as well as of the transverse linear velocities of $\mathrm{G}$ and $\mathrm{K}$ stars in latitudes above $\pm 50^{\circ}$, with a view to determining their density distribution in the direction perpendicular to the galactic plane. For this purpose he made use of the GC and the Yale repetitions of the $A G$ catalogue. He also studied the distribution of the velocities perpendicular to the galactic plane. The whole material is being used for a new determination of the force $K_{z}$.

For further work in high latitudes reference may be made to the report of the Conference on Co-ordination of Galactic Research (3r).

\section{Continuous Radiation at Radio Frequencies}

Also this subject will be dealt with in the report of another commission. I shall here confine myself to a few remarks concerning the significance of such observations for our knowledge of galactic structure.

At decimetre wave-lengths the observed radiation may largely be thermal radiation of ionized interstellar hydrogen, and will thus yield important information on the general distribution of ionized hydrogen regions throughout the Galactic System. At wavelengths between Io and $30 \mathrm{~cm}$. the resolving power obtainable with the largest parabolic aerials now in use (or soon to be taken into use) is high enough to find the true distribution over the entire system. However, at the time of writing only very incomplete measures were available.

At wave-lengths of several metres most of the radiation appears to come from radio sources. The contribution of the thermal radiation of the gas can be evaluated from the 
measures in the decimetre range and be subtracted. The effects of absorption by the ionized gas can similarly be eliminated. In order to investigate satisfactorily the remaining radiation, surveys will be required with a much higher resolution than attained up to the present. There is a promise that sufficient resolution will soon be realized by the use of so-called cross aerials. This will make it possible to decide about the portion of the non-thermal radiation having a distribution like that of the interstellar gas, and about the exact distribution of possible other contributing objects.

Related to this problem is that of the nature of the bulk of the radio sources, which has not yet been solved. Another important unsolved problem is that of a possible spherical 'corona' of radio sources around the Galactic System. There has been a tendency to ascribe the relatively high intensity of metre waves in high galactic latitudes and in the anti-centre hemisphere to radiation of extra-galactic origin, but the alternative of a major contribution from galactic sources has by no means been ruled out. Several authors (32) have even pointed at phenomena that would suggest a large spherical system of sources. This would have to differ radically from the 'spherical' distributions of optically known objects, like RR Lyrae variables or globular clusters, in that it should have an almost homogeneous distribution, with a very low density gradient.

\section{Nuclear Part and Nucleus of the Galactic System. Absolute Magnitude of RR LYRAE STARS}

The principal new results found since the writing of the previous report were the large internal motions in the interstellar gas within $3 \mathrm{kps}$ from the centre which were derived from measures of the $21 \mathrm{~cm}$. line (6). Average random radial velocities from 50 to roo $\mathrm{km}$. $/ \mathrm{sec}$. were observed in the nuclear region. From these measures estimates could also be made of the rotational velocities in this part, and of the concentration of mass toward the centre. The position of the nucleus may be estimated from these line profiles to be at $l=327^{\circ} \cdot 5, b=-I^{\circ} \cdot 5$. The density of atomic hydrogen in the nuclear region appears to be slightly lower than in our surroundings. The thickness of the gas layer is nearly the same as in the outer arms.

Measures of continuous radiation at $9 \cdot 4 \mathrm{~cm}$. (7), $21 \mathrm{~cm}$. (33) and $75 \mathrm{~cm}$. (34) wave-length all indicate, superposed upon a general increase towards the direction of the centre, a sharp concentration near the direction of the nucleus, the angular size of which appears to be smaller than the diffraction disks of the radio telescopes used. This might be a bright ordinary radio source, but its position coincides so nearly with the direction to the dynamical centre of the Galactic System that it is very tempting to assume that what is observed at these radio frequencies is the galactic nucleus itself. From the data of Haddock, Mayer and Sloanaker it follows that $l=327^{\circ} \cdot 6, b=-I^{\circ} \cdot 5$; Westerhout's measures at $2 \mathrm{I} \mathrm{cm}$. give $l=327^{\circ} 8, b=-I^{\circ} \cdot 3$, while McGee and Bolton give $327^{\circ} 9,-\mathrm{I}^{\circ} \circ$. The Washington position may be the most accurate one. The galactic nucleus might well be similar to the concentration of light near the nucleus of the Andromeda nebula.

Large velocities in the nuclear part have also been observed by Mayall and by Minkowski for the faint planetary nebulae that were found in Minkowski's and Henize's surveys.

Baade and Gaposchkin have further completed their survey of variable stars in the field at $l=329^{\circ}, b=-5^{\circ}$. They found that near the nucleus the logarithmic density gradient of the RR Lyrae variables is 0.75 per kps. They noted also a great frequency of variables with periods between 80 and I50 days. In the same field 2I3 M-type stars were found at the Warner and Swasey Observatory, indicating that in the nuclear region these stars are about four times as frequent as the RR Lyrae variables.

For two other transparent fields near the nucleus plates for variable-star surveys have been taken with the 74-inch telescope at the Radcliffe Observatory. These fields are now being investigated in Leiden.

The distance to the nucleus was found by Baade to be $8 \cdot 2 \mathrm{kps}$. The accuracy of this distance is limited by imperfect knowledge of the absorption, and by the uncertainty 
still existing in the median absolute magnitude of the RR Lyrae stars. Attempts to arrive at a better fundamental determination of this important quantity are being made in several places. Proper motions from Carte-du-Ciel plates have been derived by Pavlovskaya (35) for 80 variables; at Leiden van Herk is finishing measurements of plates taken by van Maanen and others at the Mt Wilson Observatory for about 50 variables, while a number of additional motions have been determined by Mitchell at the McCormick Observatory.

Photo-electric colours and magnitudes for all of these, as well as for a considerable number of fainter RR Lyrae variables will be determined by Kron and Eggen at the Lick Observatory.

\section{Galactic Rotation}

In this subject also important advances could be made as a consequence of the development of the $2 \mathrm{I} \mathrm{cm}$. technique. From measures made at Kootwijk Kwee, Muller and Westerhout (6) have derived velocities of rotation between 2 and $8 \mathrm{kps}$. distance from the centre. It was found that the angular velocity increases more and more steeply when proceeding towards the centre; the increase must continue to at least I kps. from the nucleus. However, not until measures for the other half of the inner system, between the southern longitudes $245^{\circ}$ and $320^{\circ}$, are also available can the rotation curve be considered to be well established. Only a comparison between the two halves can decide how closely the maximum velocities measured coincide with circular velocities.

Some deviations from circular motions had already been noted in the analysis of the gas motions in the outer part of the Galactic System, and were likewise found by Kwee, Muller and Westerhout in the longitudes $35^{\circ}$ to $45^{\circ}$ for a region within the Sun's distance from the centre.

From the work of Weaver (unpublished) and others it has become apparent that, when the constant A of differential galactic rotation is derived separately for the hemisphere around the centre and for the opposite hemisphere, the two results differ considerably. This might indicate that the run of the angular velocity $\omega$ with $R$ shows a wave in the neighbourhood of $R_{0}$ (the Sun's distance from the centre). It seems more likely, however, that we are dealing with a local deviation from the circular velocity in the region just inside $R_{\mathbf{0}}$.

\section{DyNamics}

Parenago has made an extensive study of the gravitational potential throughout the Galactic System (36). This is based principally on the distribution of RR Lyrae and other variables as found by Kukarkin. He used the following expression for the potential as function of the distance from the centre, $R$, and from the galactic plane $z$ :

$$
\Phi=\frac{\Phi_{c}}{\mathrm{I}+\kappa R^{2}} \phi(z)
$$

of which he finds that it can represent the data used in his article as well as data on longperiod cepheids previously discussed. The hydrogen rotations were not available when this investigation was made. He used his formula to compute mass densities throughout the system, and stellar orbits in the galactic plane. Such orbits have also been computed by Lohmann (37), Schütte (38) and Schwarzschild (39), all based on a very schematic model of the Galactic System.

Perek, who had previously also attempted a discussion of the RR Lyrae variables for deriving a model of the mass distribution in the Galactic System, has recently studied the orbits of the globular clusters (40), and concludes that these must in general be highly eccentric. M. Schmidt at Leiden has also made an extensive study of this subject, from which he concludes that data on density distribution are so incomplete and the number of radial velocities so limited that no reliable conclusion as to the form of the velocity distribution or the potential function of the Galactic System can be drawn from the 
globular clusters. Schmidt is using the hydrogen-line measures as well as all obtainable data on the density distribution of stars to derive a gravitational model that will fit all data available.

Lindblad and Nahon (4r) have made a detailed study of the dispersion of large star clouds in a manner similar to that used by Blaauw (42) in his investigation of the disintegration of the Scorpio-Centaurus cloud. These developments give some insight into the background of the deviation of the vertex of star-streaming and of its connexion with the manner in which the stars were once formed from interstellar clouds. They are of especial importance for the problem of the evolution of the space and velocity distribution of stars originally formed in spiral arms.

An interesting suggestion concerning the long-range evolution of the velocity distribution of stars has been developed by Spitzer and Schwarzschild (43). They have calculated the effect that passages of stars by interstellar cloud complexes may have on the velocity distribution of these stars. The result is that the increase of average random motion with advancing spectral type can be explained by this mechanism if most of the interstellar matter is gathered into cloud complexes with masses of the order of $10^{6}$ solar masses. Whether an unevenness on so large a scale is actually present, and sufficiently frequent, has not yet been proven. A quantitative treatment may become possible after a more detailed picture of the distribution of interstellar hydrogen has been obtained.

\section{Co-ordination of Galactic Research}

In the summer of 1953 a small conference was held near Groningen to study the ways in which galactic research might be co-ordinated. Before the conference a provisional report was drawn up which served as a basis for the discussions, as well as for a more extensive report in which the conclusions of the conference were collected. This final report which was drafted by Dr Blaauw who acted as secretary, has now been issued in print as the first of the series of separate symposium reports which the International Astronomical Union is going to publish. Following a proposal by the conference the Executive Committee has nominated a sub-commission of Commission 33 to deal with problems of co-ordination. The sub-commission is now constituted as follows: Baade, Blaauw, Lindblad, Oort, Parenago, and van Rhijn. It is hoped that this sub-commission will become a centre for information on current programmes, and an intermediary for exchange of ideas and suggestions wherever these are wanted by astronomers. The chairman of this sub-committee will be the chairman of Commission 33, and the secretary will be A. Blaauw.

A second committee, consisting of Baade (chairman), Kukarkin, Oosterhoff and the secretary of the first sub-commission, was similarly formed as a sub-commission of Commission 27, in order to deal with the specific task of organizing variable-star surveys.

The conference began by considering which problems appear at present to be most essential. In the second place it has discussed by which observations the solution of these problems can be promoted. Special attention was also given to desiderata for future work in the frame of the large existing co-operative undertakings: the Carte $d u$ Ciel, the Plan of Selected Areas and the Catalogue of the Astronomische Gesellschaft. Lists were drawn up of stars for which fundamental proper motions of greater precision are needed and which, therefore, require accurate meridian observations; and also, of special types of stars for which proper motions can be derived from an early repetition of Carte-du-Ciel plates.

For all further details I refer to the extensive printed report on the conference. 


\section{Proposals to be Discussed at the Meeting of Commission 33}

The following points were suggested for special discussion and eventual action by the Commission:

I. To change the name of the Commission into 'Commission on Galactic Structure' (Blaauw). I am in favour of the idea, but as a more adequate designation I would propose also to consider 'Commission on Structure and Dynamics of the Galactic System'.

2. To adopt improved standard co-ordinates of the galactic pole.

Radio observations, in particular those of the continuous radiation in the decimetre range, and those of the hydrogen line at $21 \mathrm{~cm}$., have shown that the pole of the actual plane of symmetry of the Galactic System deviates about $I: 5$ from the standard pole at $\mathrm{I} 2^{\mathrm{h}} 4 \mathrm{O}^{\mathrm{m} \cdot \mathrm{O}} ;+28^{\circ} \mathrm{O}$ (I9O0) adopted by the I.A.U. in I935 (44). This pole, on which Ohlsson's extensive tables of galactic co-ordinates are based, has been used in all recent investigations. As more and more observations are being made of distant galactic stars, for which precise galactic co-ordinates are desired, an improved standard pole has become a matter of some importance. On the other hand, the observations at radio frequencies, on which the new determination would have to rest, are at present in such a state of development that it might be wise to postpone the decision until, in one or two years, data of much greater precision will be available. Up to that time it would be advisable, in order to avoid confusion, to continue to base galactic co-ordinates on the pole mentioned, and always to use equatorial co-ordinates on the equinox I90o to compute them (so that the galactic co-ordinates of a non-moving object be constant quantities, independent of the epoch of observation).

3. Nassau proposes that the Commission recommend the establishment of infra-red sequences along the galactic equator, similar to those obtained by Kron $(A p . J$. Ir3, 324 , I95I). The LF regions observed at the Warner and Swasey Observatory, which have been chosen in relatively clear areas of the Milky Way, may prove to be particularly suitable for the purpose.

\section{J. H. OORT \\ President of the Commission}

\section{REFERENCES}

(I) W. W. Morgan, S. Sharpless and D. E. Osterbrock, Astr. J. 57, 3, 1952; see also Sky and Telescope, 11, 134, 1952.

(2) See, for example, W. W. Morgan, A. E. Whitford and A. D. Code, Ap. J. 118, 318, 1953; W. A. Hiltner, AP. J. 120, 4I, I954; B. A. Vorontsov-Velyaminov, Astr. J. U.S.S.R. 30, 37, 1953; H. F. Weaver, $A s t r . J .58,177,1953$.

(3) I. Torgård, $A p . J$. 120, 370, 1954.

(4) G. Münch, Publ. Astr. Soc. Pacif. 65, I79, 1953.

(5) H. C. van de Hulst, C. A. Muller and J. H. Oort, B.A.N. I2, I I 7, 1954.

(6) K. K. Kwee, C. A. Muller and G. Westerhout, B.A.N. I2, 2 I I, I954.

(7) F. T. Haddock, C. H. Mayer and R. M. Sloanaker, $A$ p. J. I19, 456, 1954.

(8) A. Blaauw and H. R. Morgan, B.A.N. 12, 95, 1954.

(9) P. Th. Oosterhoff, B.A.N. II, 299, I951.

(I0) B. J. Bok and U. van Wijk, Astr. J. 57, 2 I3, I952.

(I I) Trans. I.A.U. 8, 504 .

(12) See Bol. Tonantz. Tacubaya, nos. 5, 7, 8, 9, ro and I I, 1952-54;Ap.J. I18, I61, 323 and 345,1953 .

(13) W. W. Morgan, A. E. Whitford and A. D. Code, $A p . J$. I18, 318, I953.

(14) W. W. Morgan, A. B. Meinel and H. M. Johnson, $A p$. J. 120, 506, I954.

(15) W. W. Morgan, H. L. Johnson and D. Harris, $A p . J .114,523,1951$; 117, 313, I953, II8, 92, 1953; II9, I8I, 1954; 120, I96, 1954.

(16) J. J. Nassau, V. M. Blanco and W. W. Morgan, $A p$. J. x20, 478, 1954.

(17) J. J. Nassau and V. M. Blanco, $A p . J .120$, I29, I954. 
(18) J. J. Nassau and V. M. Blanco, $A p . J$. I20, 464, 1954.

(19) W. P. Bidelman, Ap. J. Suppl. no. 7, Vol. 1, I954.

(20) A. Torondzhadze, Bull. Abastumani Obs. 15, I I 5, 1953.

(21) H. F. Weaver, Publ. Astr. Soc. Pacif. 65, 132, 1953.

(22) N. G. Roman, $A p . J$. 116, I 22, 1952.

(23) A. N. Vyssotsky and A. Skumanich, Astr. J. 58, 96, 1953.

(24) J. Delhaye, C.R. 237, 294, 1953.

(25) J. Delhaye, C.R. 236, I47I, 1953.

(26) J. Kovalevsky, B.A. 18, 233, 1954.

(27) T. R. Tannahill, Mon. Not. R. Astr. Soc. 112, 3, 1952.

(28) S. W. McCuskey, $A p . J$. I15, 479, 1952; 120, 139, I954.

(29) O. Eklöf and T. Adolfsson, Medd. Astr. Obs. Uppsala, no. 106, 1953; nos. 107 and 109, 1954.

(30) C. Schalén, Medd. Astr. Obs. Uppsala, no. I08, 1954.

(3I) I.A.U. Symposium, no. I, p. $3 \mathbf{I}$.

(32) I. S. Shklovsky, Astr. J. U.S.S.R. 29,4 I8, 1952; A. D. Fokker and L. D. de Feiter, I.R.A.Rapport, no. I, I954 (in Dutch); J. E. Baldwin, Nature, Lond., 174, 320, I954.

(33) J. P. Hagen, A. E. Lilley and E. F. McClain, N.R.L. Report 4448, 1954; G. Westerhout, unpublished.

(34) R. X. McGee and J. G. Bolton, Nature, Lond., 173, 985, 1954.

(35) Partly published in Variable Stars, 9, 233, 1953.

(36) P. P. Parenago, Astr. J. U.S.S.R. 29, 245, 1952.

(37) W. Lohmann, Z. Ap. 33, I86, I953.

(38) K. Schütte, Sitz. Ber. Ak. Wien, Math.-naturw. Kl. Abt. II, 161, Heft 9-1o, 1952; 162, Heft I-5, I953; 163, Heft I-4, I954.

(39) M. Schwarzschild, Astr. J. 57, 57, 1952.

(40) L. Perek, Contr. Astr. Inst. Brno, I, no. I2, 1954.

(4I) B. Lindblad and F. Nahon, Ann. Stockholm Obs. 18, no. 2, 1954.

(42) A. Blaauw, B.A.N. II, 414, 1952.

(43) L. Spitzer and M. Schwarzschild, $A p . J .114,385,195$; 118, 106, 1953.

(44) Trans. I.A.U. 5, 375 .

\section{Report of the Meeting. 2 September 1955}

President: Prof. J. H. Oort.

SECRETARY: Dr A. Blaauw.

First, the three proposals mentioned in the Draft Report were discussed.

I. The Commission decided to propose to the Executive Committee to change its name into 'Commission on the Structure and Dynamics of the Galactic System'.

2. The second proposal, to adopt improved co-ordinates of the galactic pole, gave rise to extensive discussion. The President pointed out that, according to recent absorption measures at $21 \mathrm{~cm}$., the radio source in the direction of the galactic centre may not be identical with the galactic nucleus, but a foreground object. It should therefore not be used in adopting the position of the galactic centre, which leaves the co-ordinates of the centre less well determined than they had recently been thought to be. Baade mentioned that a 4-hr. exposure on an IN plate with the 200-inch does not show the galactic nucleus.

As more information on the position of the galactic plane may be forthcoming within the next few years, especially when southern radio surveys are more complete, a conservative attitude with regard to a revision of Ohlsson's tables is recommended, although it is clear that Ohlsson's pole is off by at least one degree. A compromise between the present necessity for new tables (urged, a.o. by Hiltner) and their postponement because of the continued improvement of the relevant observations will have to be made; the most suitable time for adopting a new galactic pole would appear to be in a year or two. The Commission discussed a suggestion by Commission 40 (Radio Astronomy) for a reso- 
lution recommending the appointment of a sub-commission to investigate the desirability of revising the galactic pole and the zero point of galactic longitude. It was suggested that the galactic centre replace the conventional zero point defined by the ascending node of the galactic circle on the equator for I900. For the final formulation of the recommendation accepted by the General Assembly see under Résolutions adoptées, where the following membership is suggested: Blaauw (convener), Pawsey and Westerhout.

It was decided that this sub-commission will not yet organize the computation of new tables. B. Lindblad announced that $\mathrm{Mr}$ Ohlsson would be interested in doing such computations. Also, names of radio astronomers who have access to large computers were mentioned.

3. In connexion with the third proposal (the establishment of infra-red sequences along the galactic equator), V. Blanco specified this, stressing the importance of the observation and publication of red and infra-red sequences to magnitude $14^{\circ} \mathrm{O}$ in the clearer areas of the Milky Way, and recommending that Commission 33 call this problem to the attention of Commission 25 (Photometry). Weaver pointed out that such standards should indeed be defined at the present time, that the wave-length region should be clearly defined, and that this matter should also be considered by the Photometry Commission. Blanco explained that the standards should be chosen along the Milky Way because there one reaches the very distant stars. Some members expressed doubt as to whether or not it would be preferable to have the standards in traditional regions (Selected Areas, etc.). Morgan pointed out that among the red stars themselves many variables occur, and Baade doubted whether it would not be better if investigators of special programmes would establish their own sequences.

As a result of the discussion, the Commission agreed on the statement that it is desirable that the standards be established in clear areas along the Milky Way.

There being no further comments on the Draft Report, it was subsequently adopted by the Commission. Discussion followed on a question raised by J. Delhaye. The Paris Observatory possesses a card catalogue of $I_{5}, 000$ stars, kept by the late $\mathrm{H}$. Mineur for the study of kinematical properties of stars for which distances and motions are known. Delhaye asked the Commission's advice on whether or not it will be desirable to keep this catalogue up to date or, perhaps, continue it on a limited basis. E. K. Kharadze mentioned that a similar card catalogue is kept at the Sternberg Institute, Moscow. W. Gliese informed the Commission that a list of stars within 20 ps with their space motions will be published next year by the Rechen-Institut. L. Gratton also has a similar card catalogue of nearby stars. W. J. Luyten and several others doubted whether it is advisable to keep the Paris catalogue up-to-date. The President pointed out that it might be useful for special, restricted programmes.

E. K. Kharadze submitted reports on work by Torondzhadze on the distribution of stellar velocities based on proper motions in galactic latitude of 3000 G.C. stars, concluding that the $z$-component distribution cannot be represented by a normal curve; work by Dsygvashvili on the galactic orbits of stars, based on Parenago's potential function; and by Kolkhidashvili on new theoretical expressions for average radial and tangential velocities based on a two-axial ellipsoid, from the application of which it is concluded that the vertex is tilted by $10^{\circ}$ with respect to the galactic equator. I. S. Shklovsky has explained the infra-red radiation of the galactic centre as due to red giants. S. A. Kaplan studied the influence of stellar encounters upon the semi-major axes and eccentricities of wide elliptical pairs, and concludes that the correlation between semi-major axis and eccentricity for the wide pairs cannot be explained by mutual encounters. 
Report on the combined meeting of Commissions 33 and 27. 2 September 1955

President: Prof. J. H. Oort.

SECRETARY: Dr A. Blaauw.

The President recalled that problems relating to the two Commissions had been discussed in I953 at the Groningen meeting on co-ordination of galactic research, and that a second meeting of similar character will probably be held in I957. W. Baade reported on the variable star survey with the 48 -inch, proposed at the Groningen conference. Test plates have been taken and will be blinked at Groningen. Next year, L. Plaut will work on this programme at Palomar. The President mentioned the necessity of supplementing this programme with observations of colour excesses and radial velocities of the nearer variable stars.

B. V. Kukarkin reported on the Soviet surveys of variable stars, and requested the collaboration of Harvard and Sonneberg on a project of re-investigating all known variables brighter than the I2th magnitude, in which Stalinabad Observatory is engaged. The Commission adopted a resolution proposed by B. V. Kukarkin, recommending such a collaboration (see Résolutions adoptées). At Harvard, H. Shapley was reported to work on objects in the galactic halo and near the galactic pole, and Miss D. Hoffleit on the Sagittarius region. J. J. Nassau remarked that spectra for all the red variables found in the 48 -inch survey can be taken at Cleveland. C. Hoffmeister reported on work at Sonneberg; the Milky Way belt from Scutum to Puppis is under investigation and special attention is given to the short-period variables. Leiden Observatory reported continuing the variable-star survey in the southern Milky Way.

The President next referred to the Milky Way test regions described in the Groningen Report for the determination of the space distribution of Ao stars and $\mathrm{K}$ giants. B. Lindblad reported that plates taken for this purpose by Nassau will be measured at Stockholm. He also called attention to work by J. Ramberg, who summarized his spectrophotometric work on stars down to photographic magnitude 13.5. The survey reaches out to distances of 3000 ps and shows for class Ao stars an increase in density at the location of the Perseus arm. J. J. Nassau reported on S. W. McCuskey's work on $\mathrm{B} 8$ to A2 stars brighter than magnitude $\mathrm{I} 2 \cdot 3$ between galactic longitudes $140^{\circ}$ and $\mathrm{I} 70^{\circ}$, which shows a density increase between rooo and 2000 ps., a region where also the radio results indicate high density of neutral hydrogen.

Finally, some special problems were discussed. B. V. Kukarkin stressed the importance of using old Carte du Ciel plates for proper-motion work on variables and urged observatories which are suitably equipped to take part in the observation and measurements of proper motions for the Plaut list of variable stars. The President reported work on proper motions of RR Lyrae stars by G. van Herk at Leiden. Bidelman stated that it is planned at Lick to observe faint variable stars for radial velocity, especially those of the cepheid and.mira type. The Chairman suggested that the RR Lyrae type stars were also of importance in this field of research and pointed out that their luminosities could be regarded as better known than those of the Mira-type stars. W. Baade recommended a search for long-period variables in the Magellanic Clouds on pv plates. L. Gratton reported on spectrographic observations of cepheids south of $-20^{\circ}$ and brighter than 9th magnitude at maximum. The President quoted from a letter by L. Perek who drew attention to the importance of studying stars with hyperbolic orbits. Miss N. Roman reported on work in high-latitude Selected Areas; slit spectra for luminosity classification were taken in areas north of $50^{\circ}$ latitude. Radial velocities of these stars, if brighter than 9.5 , are being measured at Toronto. The President reported on work on the distances of southern cepheids, based on new colour measures by P. Th. Oosterhoff and Th. Walraven. Bidelman reported on similar work by Eggen in the northern hemisphere, indicating that the cepheids are located in the spiral arm, but not correlated with the associations. 